\title{
Autoimmune hyperphosphatemic tumoral calcinosis in a patient with FGF23 autoantibodies
}

\author{
Mary Scott Roberts, ${ }^{1}$ Peter D. Burbelo, ${ }^{2}$ Daniela Egli-Spichtig, ${ }^{3}$ Farzana Perwad, ${ }^{3}$ Christopher J. Romero, ${ }^{4}$ Shoji Ichikawa, ${ }^{5}$ \\ Emily Farrow, ${ }^{6}$ Michael J. Econs, ${ }^{5,7}$ Lori C. Guthrie, ${ }^{1}$ Michael T. Collins, ${ }^{1}$ and Rachel I. Gafni ${ }^{1}$ \\ 'Skeletal Disorders and Mineral Homeostasis Section, and '2Dental Clinical Research Core, National Institute of Dental and Craniofacial Research (NIDCR), NIH, Bethesda, Maryland, USA. ${ }^{3}$ Department of \\ Pediatrics, Division of Nephrology, UCSF School of Medicine, San Francisco, California, USA. ${ }^{4}$ Department of Pediatric Endocrinology and Diabetes, Icahn School of Medicine at Mount Sinai, New York, \\ New York, USA. '5 Department of Medicine, Indiana University School of Medicine, Indianapolis, Indiana, USA. ' ${ }^{6}$ Center for Pediatric Cenomic Medicine, Children's Mercy Hospital, Kansas City, Missouri, USA. \\ ${ }^{7}$ Department of Medical and Molecular Genetics, Indiana University School of Medicine, Indianapolis, Indiana, USA.
}

\begin{abstract}
Hyperphosphatemic familial tumoral calcinosis (HFTC)/hyperostosis-hyperphosphatemia syndrome (HHS) is an autosomal recessive disorder of ectopic calcification due to deficiency of or resistance to intact fibroblast growth factor 23 (iFGF23). Inactivating mutations in FGF23, $\mathrm{N}$-acetylgalactosaminyltransferase 3 (GALNT3), or KLOTHO (KL) have been reported as causing HFTC/HHS. We present what we believe is the first identified case of autoimmune hyperphosphatemic tumoral calcinosis in an 8-year-old boy. In addition to the classical clinical and biochemical features of hyperphosphatemic tumoral calcinosis, the patient exhibited markedly elevated intact and C-terminal FGF23 levels, suggestive of FGF23 resistance. However, no mutations in FGF23, KL, or FGF receptor 1 (FGFR1) were identified. He subsequently developed type 1 diabetes mellitus, which raised the possibility of an autoimmune cause for hyperphosphatemic tumoral calcinosis. Luciferase immunoprecipitation systems revealed markedly elevated FGF23 autoantibodies without detectable FGFR1 or Klotho autoantibodies. Using an in vitro FGF23 functional assay, we found that the FGF23 autoantibodies in the patient's plasma blocked downstream signaling via the MAPK/ERK signaling pathway in a dose-dependent manner. Thus, this report describes the first case, to our knowledge, of autoimmune hyperphosphatemic tumoral calcinosis with pathogenic autoantibodies targeting FGF23. Identification of this pathophysiology extends the etiologic spectrum of hyperphosphatemic tumoral calcinosis and suggests that immunomodulatory therapy may be an effective treatment.
\end{abstract}

\section{Introduction}

Fibroblast growth fact 23 (FGF23) is a phosphate- and 1,25(OH) ${ }_{2}$ vitamin D-regulating (1,25D-regulating) hormone produced by osteoblasts and osteocytes (1). FGF23 acts via FGF receptor 1 (FGFR1) coupled with the coreceptor $\alpha$ Klotho to reduce expression of sodium phosphate cotransporters (NaPi-2a and -2c) and renal 25-hydroxy vitamin D 1- $\alpha$-hydroxylase (2). FGF23 lowers serum phosphorus and $1,25 \mathrm{D}$ levels by its actions on the kidney to reduce renal tubular reabsorption of phosphate (TRP) and 1,25D production. Excess FGF23 has been implicated in a number of hypophosphatemic disorders, such as tumor-induced osteomalacia (3), X-linked hypophosphatemia (4), and autosomal dominant hypophosphatemic rickets (5).

Hyperphosphatemic familial tumoral calcinosis (HFTC)/ hyperostosis-hyperphosphatemia syndrome (HHS) (OMIM

Conflict of interest: The NIDCR has received financial support from Shire, Novartis, and BridgeBio for research using pharmaceutical agents not discussed in this work. MSR became an employee of Ultragenyx Pharmaceutical in September 2017, after work on this project was completed. Indiana University has licensed FGF23 to Kyowa Hakko Kirin Co., and MJE receives royalty income through this license. MJE serves as a consultant to Ultragenyx Pharmaceutical and receives research support from Horizon Pharma for an unrelated trial.

License: Copyright 2018, American Society for Clinical Investigation.

Submitted: May 7, 2018; Accepted: September 11, 2018.

Reference information: J Clin Invest. 2018;128(12):5368-5373.

https://doi.org/10.1172/JCl122004.
211900) is a disorder of FGF23 deficiency or resistance. Affected individuals develop ectopic calcifications called tumoral calcinosis and/or diaphyseal hyperostosis, which manifests clinically in the long bones as diaphyseal pain and swelling (6). Characteristic dental findings of HFTC/HHS include shortened roots with dilacerations, thistle-shaped dental pulps, pulp chamber and root canal obliteration, and pulp stones (7). In addition, some patients experience systemic inflammation.

HFTC/HHS is an autosomal recessive disease, and to date, causal mutations in 3 genes have been identified: FGF23 (12p13.3) (8), UDP-GalNAc:polypeptide $N$-acetylgalactosaminyltransferase 3 (GALNT3) (2q24-q31) (9), and Klotho (KL) (13q12) (10). Mutations in FGF23 and GALNT3 result in premature cleavage of biologically active intact FGF23 (iFGF23) into inactive fragments, while mutations in $K L$ interrupt FGF23 signaling, causing FGF23 resistance. Lack of iFGF23 results in hyperphosphatemia, due to increased TRP and elevated or inappropriately normal 1,25D production, which promotes gastrointestinal absorption of phosphorus and calcium. The net effect is an increase in the calcium $x$ phosphate product, leading to tumoral calcinosis.

Here, we present what we believe is the first case of autoimmune hyperphosphatemic tumoral calcinosis due to pathogenic FGF23 autoantibodies. Autoantibodies directed against extracellular targets, such as receptors (e.g., nicotinic acetylcholine receptor autoantibodies in myasthenia gravis) or secreted mole- 
Table 1. Biochemical results in patient with autoimmune tumoral calcinosis

\begin{tabular}{|c|c|c|c|c|}
\hline & $\begin{array}{c}6 \mathrm{yr}, 7 \mathrm{mo} \text { (initial } \\
\text { laboratory evaluation) }\end{array}$ & $7 \mathrm{yr}, 3 \mathrm{mo}$ & $7 \mathrm{yr}, 9 \mathrm{mo}$ & $8 \mathrm{yr}$ \\
\hline Treatment & & SEV & SEV, ACZ & SEV, ACZ \\
\hline Phosphorus (3.2-6.3 mg/dl) & 7.2 & 7.9 & 6.6 & 5.6 \\
\hline Calcium (8.2-10 mg/dl) & 9.9 & 9.9 & 9.7 & 10.1 \\
\hline Calcium $\times$ phosphorus $\left(<65 \mathrm{mg}^{2} / \mathrm{dl}^{2}\right.$ for $\left.<12 \mathrm{yr}\right)$ & 71 & 78 & 64 & 56 \\
\hline $1,25-(\mathrm{OH})_{2}$ Vitamin $\mathrm{D}_{3}(24-86 \mathrm{pg} / \mathrm{ml})$ & & 84 & & 59 \\
\hline Intact PTH (15-65 pg/ml) & & 21 & & 11.2 \\
\hline $\operatorname{TRP}(85 \%-95 \%)$ & & 95 & 97.2 & 93 \\
\hline TP/GFR (age specific mean 4.4 mg/dl; ref. 24) & & 6.1 & 6.5 & 5.2 \\
\hline iFGF23 (<52 pg/ml) & 13,000 & 7800 & & 6900 \\
\hline CFGF23 (3M-17Y $\leq 230)$ & 33,000 & 33,000 & & 22,400 \\
\hline $\operatorname{CRP}(<4.99 \mathrm{mg} / \mathrm{l})$ & & 1.7 & & $<0.15$ \\
\hline $\operatorname{ESR}(0-42 \mathrm{~mm} / \mathrm{h})$ & & 37 & & 12 \\
\hline Glucose (nonfasting < $200 \mathrm{mg} / \mathrm{dl}$ ) & 82 & 119 & $\begin{array}{c}81 \\
\text { (fasting) }\end{array}$ & 433 \\
\hline Hemoglobin A1c (4\%-6\%) & & & & 10.7 \\
\hline Insulin (2.6-24.9 mcU/ml) & & & & 4.1 \\
\hline C-peptide (1.1-5 ng/ml) & & & & 0.8 \\
\hline Bicarbonate (22-29 mmol/l) & & 28 & 20 & 23 \\
\hline
\end{tabular}

ACZ, acetazolamide; SEV, sevelamer; PTH, parathyroid hormone; TP, tubular maximum reabsorption of phosphorus; GFR, glomerular filtration rate; CRP, C-reactive protein; ESR, erythrocyte sedimentation rate.
At 7 years, 3 months, the patient was referred to the NIH, and acetazolamide was added to promote renal phosphate excretion. After 9 months on this regimen, the right hip tumoral calcinosis was decreased in size on physical exam and repeat radiograph (Figure 1, A-D) and no new lesions had developed. The blood phosphorus fluctuated, but decreased overall, with a nadir of $5.4 \mathrm{mg} / \mathrm{dl}$ (Supplemental Figure 1; supplemental material available online with this article; https://doi.org/10.1172/ JCI122004DS1).

Given the considerably elevated iFGF23 and CFGF23 levels in this patient compared with reported cases of HFTC/HHS and lack of an identifiable genetic cause, we questioned whether an interfering antibody led to falsely elevated iFGF23 and CFGF23 levels. Therefore, we cules (e.g., granulocyte-macrophage-stimulating factor autoantibodies in pulmonary alveolar proteinosis), have been previously described as causing human disease (11). In this case, the patient exhibited classic features of HFTC/HHS: tumoral calcinosis with hyperphosphatemia in the setting of normal renal function and increased TRP. iFGF23 levels were high, suggesting FGF23 resistance. However, a causative genetic mutation was not identified. After the patient subsequently developed type 1 diabetes mellitus (T1DM), an autoimmune disease, luciferase immunoprecipitation systems (LIPS) (12) were used to detect potential autoantibodies against proteins in the FGF23-signaling receptor complex. An in vitro FGF23 functional assay was performed to assess the effect of FGF23 autoantibodies on FGF23 signal transduction.

\section{Results and Discussion}

This White boy presented at 6 years, 3 months, with pain, swelling, and development of a firm lesion on the lateral right hip. MRI of the lesion revealed a calcified mass in the right gluteus maximus extending into the subcutaneous soft tissue. A biopsy of the lesion showed tumoral calcinosis. Subsequent laboratory evaluation revealed hyperphosphatemia $(7.2 \mathrm{mg} / \mathrm{dl}$; normal for age, 3.2-6.3) with normal blood calcium and renal function, consistent with the diagnosis of HFTC/HHS. iFGF23 and C-terminal FGF23 (CFGF23) levels were markedly elevated, at $13,000 \mathrm{pg} / \mathrm{ml}$ (nor$\mathrm{mal},<52$ ) and 33,000 RU/ml (normal for age, $\leq 230$ ) (Table 1), respectively, consistent with FGF23 resistance. The patient was started on the phosphate binder sevelamer and a low-phosphate diet. Sequencing of FGF23, KL, and FGFR1 genes and wholeexome sequencing did not identify gene mutations or genetic variants, including mutations and/or variants in GALNT3, that could explain the clinical calcinosis/phosphate phenotype. performed serial dilutions of the patient's plasma (1:1, 1:10, 1:20, 1:50, 1:100, 1:500, 1:1000) and measured iFGF23 and CFGF23 via second-generation ELISA kits (Immutopics). For both iFGF23 and CFGF23, levels decreased with serial dilution as expected, except for the 1:500 and 1:1000 dilutions of iFGF23, which were below the lower limit of detection of the assay (Supplemental Table 1). The ability to detect dilutions of iFGF23 and CFGF23 via ELISA ruled out the presence of an interfering antibody resulting in the elevated iFGF23 and CFGF23 levels.

Twenty months after initial symptom onset, the patient reported a 2-week history of polyuria, polydipsia, and nocturia, with no change in appetite or weight. He denied dysuria, hematuria, enuresis, or fever. Laboratory evaluation revealed blood phosphorus of $5.6 \mathrm{mg} / \mathrm{dl}$ (normal for age, 3-5.7), 1,25D of $59 \mathrm{pg} / \mathrm{ml}$ (normal, 24-86), and TRP of 93\% (normal, $85 \%-95 \%$ in the setting of normal blood phosphorus). iFGF23 and CFGF23 levels remained markedly elevated at $6900 \mathrm{pg} / \mathrm{ml}$ (normal, <52) and 22,400 RU/ $\mathrm{ml}$ (normal for age $\leq 230$ ), respectively. In addition, the subject was hyperglycemic (fasting blood glucose, $433 \mathrm{mg} / \mathrm{dl}$; normal, < 120) and insulinopenic $(4.1 \mathrm{mcU} / \mathrm{ml}$; normal fasting, 2.6-24.9) with a low C-peptide (0.8 ng/ml; normal, 1.1-1.5). Hemoglobin A1c was elevated at $10.7 \%$ (estimated average glucose, $260 \mathrm{mg} / \mathrm{dl}$ ) (Table 1). The subject had positive islet antigen-2 (IA-2) and antiinsulin antibodies and negative glutamic acid decarboxylase (GAD65) antibodies. Of note, he had a normal fasting blood glucose of 81 $\mathrm{mg} / \mathrm{dl} 3$ months prior. These results were consistent with newonset T1DM. The patient was admitted for initiation of insulin therapy and diabetes education.

Given the lack of an identifiable genetic cause for hyperphosphatemic tumoral calcinosis and the new diagnosis of T1DM, we hypothesized that the tumoral calcinosis could be autoimmune 


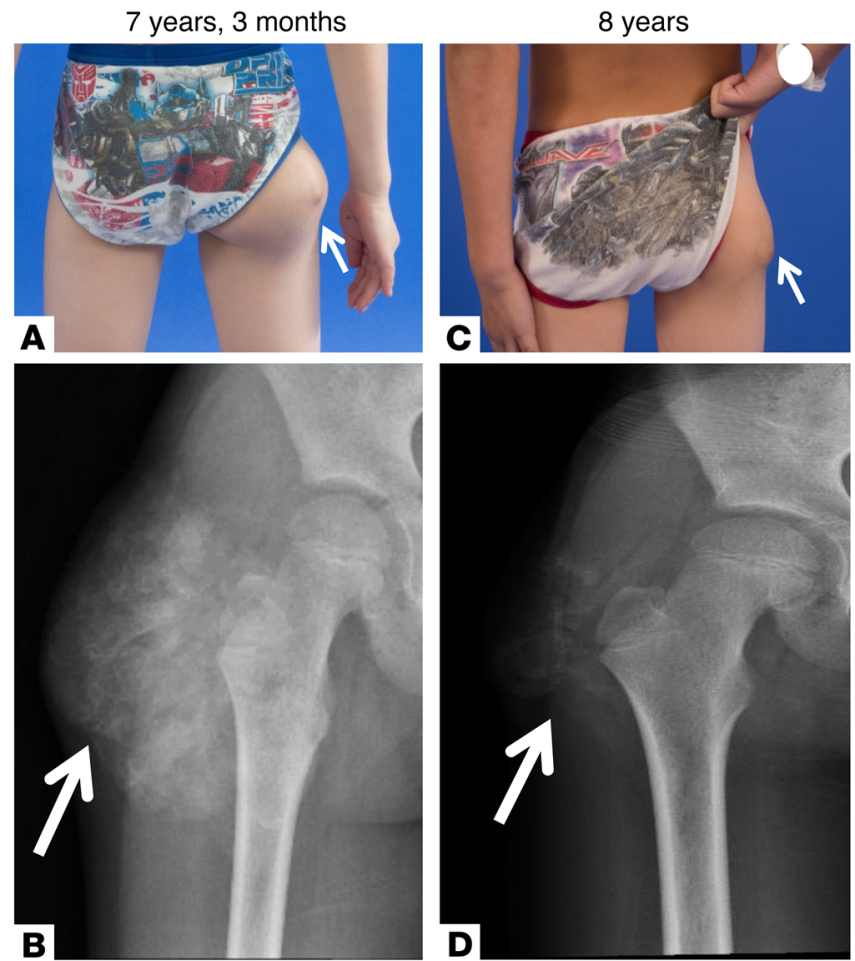

in nature. Based on previously described LIPS tests for IA-2 (13) and GAD65 (14) autoantibodies, our patient demonstrated seropositive autoantibodies against IA-2 (Figure 2A) and seronegative autoantibodies to GAD65 (Figure 2B), consistent with results obtained at diagnosis of T1DM. In order to test the hypothesis that autoantibodies might be directed against extracellular components of the FGF23-signaling pathway, light-emitting luciferase fusion proteins for FGF23, FGFR1, and Klotho were generated for LIPS autoantibody profiling (12). Remarkably, the patient showed very high levels of autoantibodies against the FGF23-Gaussia luciferase fusion protein. These were 60 -fold higher than those of other patients with HFTC due to mutations in GALNT3 (Figure $2 \mathrm{C})$. Moreover, patients with a variety of other autoimmune diseases including Sjögren syndrome, systemic lupus erythematosus, vasculitis, and T1DM, were all seronegative for FGF23 autoantibodies (Figure 2C). No significant detectable immunoreactivity was found against FGFR1 (Figure 2D) and Klotho (Figure 2E), as the autoantibody levels in the patient were comparable to those of other patients with HFTC and healthy controls. These findings demonstrate the patient had elevated levels of autoantibodies that were selectively against FGF23, but not against the corresponding receptor proteins for this ligand. Because FGF23 autoantibody titers were only measured at 2 time points, a potential correlation between autoantibody levels and blood phosphorus, FGF23, and $1,25 \mathrm{D}$ could not be evaluated.

While we have not mapped the regions of FGF23 reacting with the patient's autoantibodies, it is likely that the patient harbors a complex mixture of autoantibodies that target both linear and conformational epitopes of FGF23 and block the action of FGF23 on its receptor. Interestingly, the patient's autoantibodies did not interfere with the iFGF23 or CFGF23 ELISA assays. This is likely explained by the fact that the antibodies used in the
Figure 1. Clinical presentation and radiographs of patient with tumoral calcinosis. (A) Tumoral calcinosis (arrows) of the lateral right hip at initial presentation to the NIH at 7 years, 3 months old. (B) Radiograph of the lesion revealed a soft tissue mass with amorphous calcifications around the right greater trochanter consistent with tumoral calcinosis. Repeat evaluation 9 months after initial presentation while on phosphate-lowering medications (sevelamer and acetazolamide) and a low-phosphate diet showed decrease in the size of the tumoral calcinosis (arrows) on physical exam (C) and repeat radiograph (D).

ELISAs are generated against relatively small, defined polypeptides derived from the 2 ends of the FGF23 protein and would not be influenced by the existing patient's autoantibodies. Studies with other pathogenic autoantibodies, including pulmonary alveolar proteinosis and membranous nephropathy, have also used similar antibody-based methods to establish that the protein levels of the granulocyte-macrophage CSF (GM-CSF) (15) and anti-phospholipase A2 receptor (PLA2R) (16), respectively, are unchanged or increased in these autoimmune diseases. Finally, our concordant findings from 2 different commercial ELISA assays showing comparable results with elevated FGF23 protein levels potentially support the validity of this approach.

Based on the elevated FGF23 autoantibodies found in the patient, we tested the effect of FGF23 autoantibodies on FGF23 signaling in an in vitro functional assay. Human embryonic kidney cells (HEK293) stably transfected with the full-length transmembrane form of mouse Klotho (17) were treated with vehicle (PBS), recombinant human FGF23 R176Q, or basic FGF (bFGF) in the presence of either $2 \%$ plasma from our patient or $2 \%$ human control plasma. Recombinant FGF23 increased early growth response 1 (EGR1) mRNA expression in a dose-dependent manner, whereas the patient's plasma blocked the FGF23-dependent increase in EGR1 mRNA expression. The inhibitory effect of the patient's plasma on EGR1 mRNA expression was overcome with the addition of $100 \mathrm{ng} / \mathrm{ml} \mathrm{FGF} 23$ (Figure 3A). Control plasma had no effect on EGR1 mRNA expression at any FGF23 concentration (Figure 3B). Recombinant bFGF increased EGR1 mRNA expression independently of the patient's plasma (Figure 3C), indicating that autoantibodies present in our patient's plasma are specific in blocking the action of FGF23.

FGF23 binding to the FGFR1-Klotho receptor complex leads to ERK1/2 phosphorylation. We analyzed FGF23-depen- 

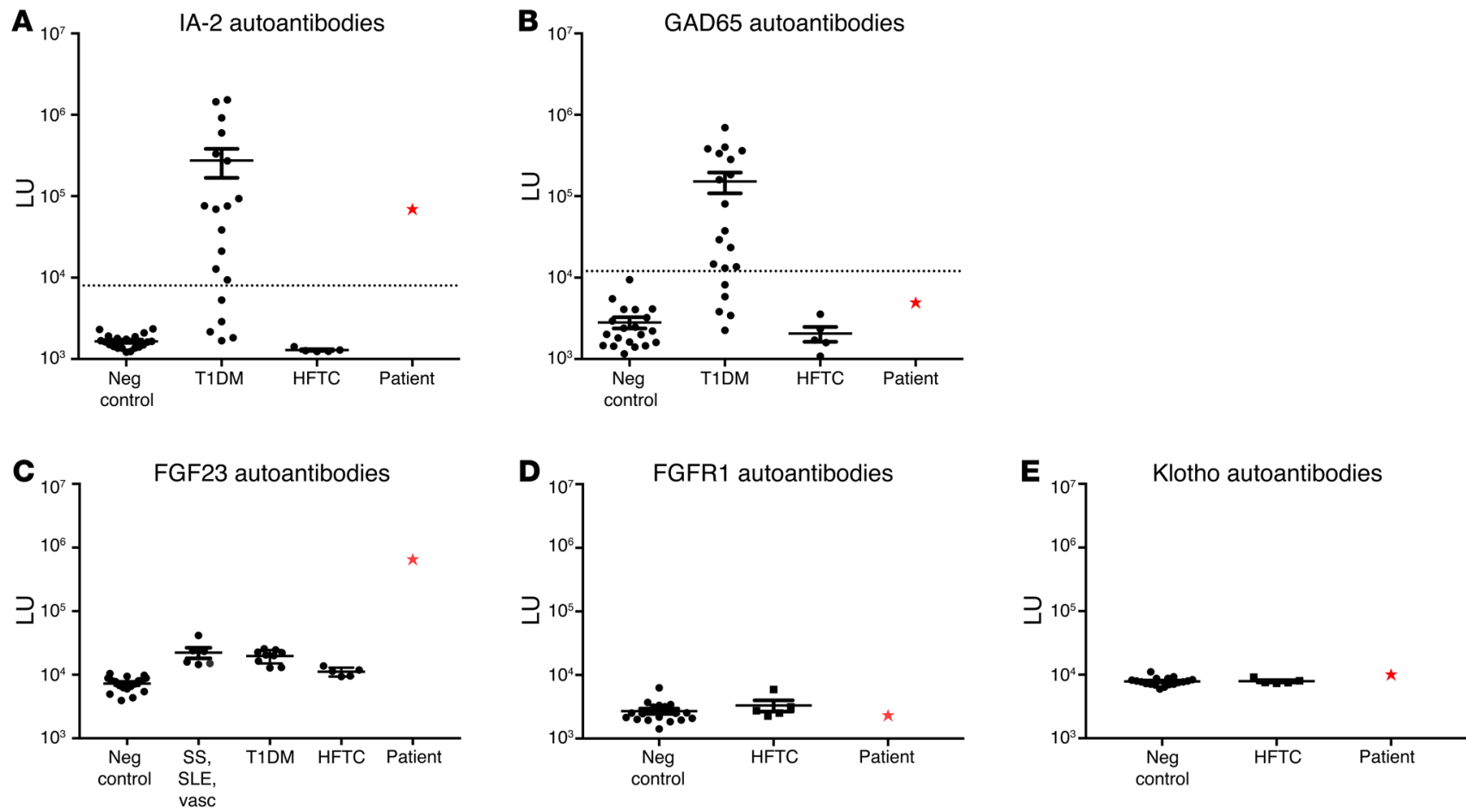

Figure 2. LIPS detection of autoantibodies. LIPS evaluation revealed the patient (red star) had seropositive autoantibodies against IA-2 (A), consistent with the diagnosis of T1DM, and seronegative autoantibodies to GAD65 (B). The patient showed very elevated levels of autoantibodies against FGF23 that were 60 -fold higher than those of other patients with HFTC/HHS. The patient also had substantially higher levels of FGF23 autoantibodies compared with healthy controls and other patients with a variety of autoimmune diseases. SS, Sjögren's syndrome; SLE, systemic lupus erythematosus; vasc, vasculitis. (C). The patient (red star) had levels of autoantibodies to FGFR1 (D) and Klotho (E) that were similar to those of other patients with HFTC and healthy controls. Dotted lines show mean light units (LU) value plus 3 SDs of the serum samples from healthy controls (14). Neg, negative.

dent phosphorylation of ERK1/2 in HEK293-Klotho cells in the presence or absence of patient or control plasma. Recombinant FGF23 significantly increased ERK1/2 phosphorylation, which was blocked by the patient's plasma (Figure 3, D and G), suggesting that the patient's plasma blocks FGF23 binding to its receptor. Control plasma did not interfere with ERK1/2 phosphorylation (Figure 3, E and H). Furthermore, bFGF increased ERK1/2 phosphorylation, but the patient's plasma had no inhibitory effect on ERK1/2 phosphorylation upon bFGF treatment (Figure 3, F and I). Changes in EGR1 protein levels were not assessed in the in vitro assay, given that the patient's plasma with FGF23 was shown to block both EGR1 expression and ERK1/2 phosphorylation, which is not seen with control plasma/FGF23 or the patient's plasma/bFGF. This is comparable to what has been shown in the Hyp mouse, which, when treated with an ERK1/2 inhibitor, had decreased FGF23 signaling and increased serum phosphorus, $1,25 \mathrm{D}$, and skeletal mineralization $(18,19)$.

Given the onset of 2 autoimmune diseases within 2 years, we examined the patient's autoimmune and immunological characteristics. Lymphocyte immunophenotyping using flow cytometry was performed. The patient had an overall normal cell profile with normal plasmablasts and total B cells with typical representation of immature, transitional, and memory B cell subsets. He had low levels of the autoreactive $\mathrm{CD} 21^{\text {lo }} \mathrm{CD} 38^{\text {lo }} \mathrm{B}$ cell subset (20), which is enriched in patients with autoimmune polyendocrinopathy-candidiasis-ectodermal dystrophy (APECED) (21), common variable immunodeficiency-associated autoimmune cytopenias (22), and cytotoxic T lymphocyte-associated protein 4 (CTLA4) haploinsufficiency-associated immune dysregulation (23). Evaluation of T and B cell clonality was performed via PCR amplified for detection of immunoglobulin (IgH and IgK loci) and $\mathrm{T}$ cell receptor gene rearrangements. No significant $\mathrm{T}$ or $\mathrm{B}$ cell populations were identified. The subject was screened for autoantibodies associated with other autoimmune diseases and did not demonstrate autoantibodies to thyroglobulin, thyroid peroxidase, tissue transglutaminase, intrinsic factor, parietal cells, and 21-hydroxylase. He had normal immunoglobulin (IgG, IgA, and IgM) levels. Although the exact mechanism involved in the generation of anti-FGF23 autoantibodies in this subject is unknown, we speculate that autoreactive B cells not identified in the immune cell evaluation and/or epitope spreading in the context of T1DM might be responsible. Given the lack of an identifiable target for treatment, immunomodulatory medications were not recommended for this patient.

Here, we describe what we believe is the first case of pathogenic autoantibodies that target FGF23, causing hyperphosphatemic tumoral calcinosis. This observation is supported by identification of elevated levels of FGF23 autoantibodies, which, in vitro, block FGF23 action at the level of the FGFR1-Klotho receptor complex. We speculate that the FGF23 autoantibodies likely sequester FGF23 and prevent it from interacting with its receptor to promote normal signal transduction. The present data depict a pathophys- 

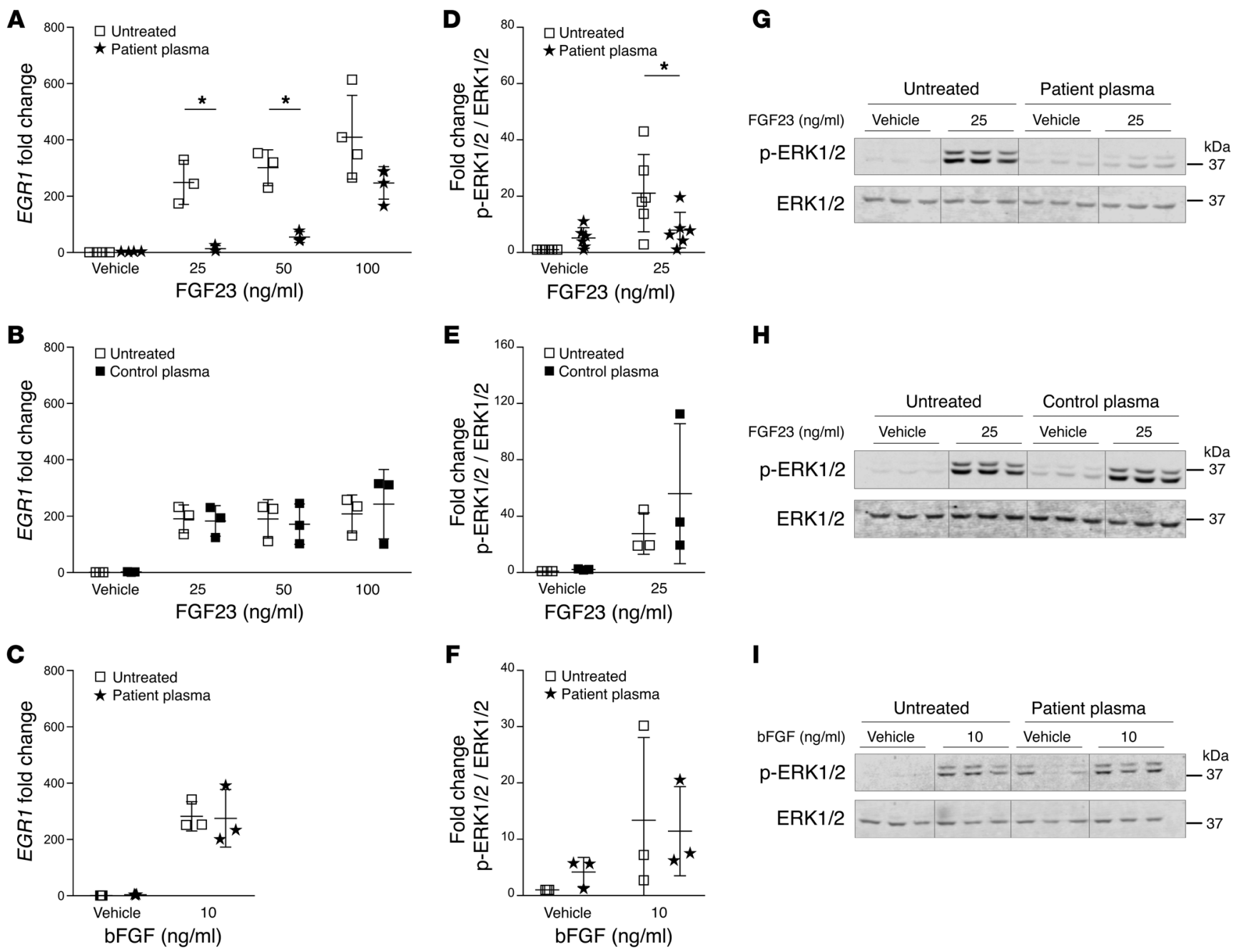

Figure 3. The effect of patient plasma on FGF23 signaling in HEK293-Klotho cells. Relative EGR1 mRNA expression compared with 18S rRNA in HEK293Klotho cells treated for 2 hours with either recombinant human FGF23 (vehicle, 25, 50, or $100 \mathrm{ng} / \mathrm{ml}$ ) (A and B) or recombinant human bFGF (vehicle or $10 \mathrm{ng} / \mathrm{ml})(\mathbf{C})$ and $\pm 2 \%$ patient plasma (A and $\mathbf{C}$ ) or $\pm 2 \%$ control plasma (B). $n=3$. Single independent experiments were normalized to their untreated controls. Fold change of phosphorylated ERK1/2 (p-ERK1/2) protein abundance compared with ERK1/2 in HEK293-Klotho cells treated for 15 minutes with either vehicle, recombinant human FGF23 $(25 \mathrm{ng} / \mathrm{ml})(\mathbf{D}, \mathbf{E}, \mathbf{G}, \mathbf{H})$, or recombinant human bFGF $(10 \mathrm{ng} / \mathrm{ml})(\mathbf{F}$ and $\mathbf{I})$ and $\pm 2 \%$ patient plasma $(\mathbf{D}, \mathbf{F}, \mathbf{G}, \mathbf{I})$ or $\pm 2 \%$ control plasma (E and $\mathbf{H}) . n=3-6$. Single independent experiments were normalized to vehicle control. Representative blots of single experiments show decreased p-ERK1/2 protein abundance with recombinant FGF23 $(25 \mathrm{ng} / \mathrm{ml})$ compared with vehicle-treated cells in the presence of patient's plasma (C). Addition of control plasma in the presence of FGF23 resulted in no change in p-ERK1/2 protein abundance (H). There was no change in p-ERK1/2 protein abundance with bFGF $(10 \mathrm{ng} / \mathrm{ml})$ in the presence of patient's plasma (I). Data are presented as mean + SD, and 2-way ANOVA followed by Tukey's test for multiple comparisons was used for statistical analysis. ${ }^{*} P<0.05$.

iology for hyperphosphatemic tumoral calcinosis and suggest a potential role for immunomodulatory therapy in the treatment of this disease if an immunological target can be identified.

\section{Methods}

Further information is provided in Supplemental Methods.

Statistics. The statistical analyses were performed using 2-way ANOVA, followed by Tukey's test for multiple comparisons (GraphPad Prism, version 7.02). Data are provided as mean +SD. A P value of less than 0.05 was considered statistically significant.

Study approval. This study was approved by the Institutional Review Board of the NIDCR (NIH). The parents of the subject provided written consent to the study protocol, and the subject provided written assent.

\section{Author contributions}

RIG and MTC oversaw the study. MSR, RIG, and MTC coordinated the work and prepared the manuscript, which was reviewed, edited and approved by all authors. MSR, CJR, LCG, MTC, and RIG clinically examined the patient, collected blood samples, and prescribed medical treatment. SI, EF, and MJE performed DNA sequencing, genetic analyses, and whole-exome sequencing. PDB performed LIPS experiments. DES and FP performed in vitro assay work.

\section{Acknowledgments}

We thank the patient and his family for participating in this study. Thanks to Mihalis S. Lionakis for analyzing the flow cytometry results. This research was supported, in part, by the Division of 
Intramural Research, NIDCR, a part of the Intramural Research Program of the NIH, Department of Health and Human Services (to MSR, PDB, LCG, MTC, RIG). Additional grant support was as follows: R21 AR068615 and RO1 AR42228 (to MJE); Swiss National Science Foundation grant no. 161989 (to DES).
Address correspondence to: Rachel I. Gafni or Michael T. Collins, Section on Skeletal Disorders and Mineral Homeostasis, NIDCR, NIH, Building 30, Room 228, 30 Convent Drive, MSC 4320, Bethesda, Maryland 20892-4320, USA. Phone: 301.594.9924; Email: gafnir @nih.gov (R.I. Gafni); mcollins@dir.nidcr.nih.gov (M.T. Collins).
1. Riminucci M, et al. FGF-23 in fibrous dysplasia of bone and its relationship to renal phosphate wasting. J Clin Invest. 2003;112(5):683-692.

2. Shimada T, et al. FGF-23 is a potent regulator of vitamin D metabolism and phosphate homeostasis. J Bone Miner Res. 2004;19(3):429-435.

3. Chong WH, Molinolo AA, Chen CC, Collins MT. Tumor-induced osteomalacia. Endocr Relat Cancer. 2011;18(3):R53-R77.

4. Jonsson KB, et al. Fibroblast growth factor 23 in oncogenic osteomalacia and X-linked hypophosphatemia. NEngl JMed. 2003;348(17):1656-1663.

5. White KE, Carn G, Lorenz-Depiereux B, Benet-Pages A, Strom TM, Econs MJ. Autosomaldominant hypophosphatemic rickets (ADHR) mutations stabilize FGF-23. Kidney Int. 2001;60(6):2079-2086.

6. Narchi H. Hyperostosis with hyperphosphatemia: evidence of familial occurrence and association with tumoral calcinosis. Pediatrics. 1997;99(5):745-748.

7. Dumitrescu CE, et al. A case of familial tumoral calcinosis/hyperostosis-hyperphosphatemia syndrome due to a compound heterozygous mutation in GALNT3 demonstrating new phenotypic features. Osteoporos Int. 2009;20(7):1273-1278.

8. Benet-Pagès A, Orlik P, Strom TM, LorenzDepiereux B. An FGF23 missense mutation causes familial tumoral calcinosis with hyperphosphatemia. Hum Mol Genet. 2005;14(3):385-390.

9. Topaz O, et al. Mutations in GALNT3, encoding a protein involved in O-linked glycosylation, cause familial tumoral calcinosis. Nat Genet. 2004;36(6):579-581.

10. Ichikawa S, et al. A homozygous missense mutation in human KLOTHO causes severe tumoral calcinosis. JMusculoskelet Neuronal Interact. 2007;7(4):318-319.

11. Burbelo PD, Keller J, Kopp JB. New horizons for human pathogenic autoantibodies. Discov Med. 2015;20(108):17-25.

12. Burbelo PD, Lebovitz EE, Notkins AL. Luciferase immunoprecipitation systems for measuring antibodies in autoimmune and infectious diseases. Transl Res. 2015;165(2):325-335.

13. Burbelo PD, et al. A new luminescence assay for autoantibodies to mammalian cell-prepared insulinoma-associated protein 2. Diabetes Care. 2008;31(9):1824-1826.

14. Burbelo PD, et al. Comparison of radioimmunoprecipitation with luciferase immunoprecipitation for autoantibodies to GAD65 and IA-2beta. Diabetes Care. 2010;33(4):754-756.

15. Uchida K, et al. High-affinity autoantibodies specifically eliminate granulocyte-macrophage colony-stimulating factor activity in the lungs of patients with idiopathic pulmonary alveolar proteinosis. Blood. 2004;103(3):1089-1098.

16. Debiec H, Ronco P. PLA2R autoantibodies and PLA2R glomerular deposits in membranous nephropathy. N Engl JMed. 2011;364(7):689-690.

17. Kurosu H, et al. Regulation of fibroblast growth factor-23 signaling by klotho. J Biol Chem . 2006;281(10):6120-6123.

18. Ranch D, Zhang MY, Portale AA, Perwad F. Fibroblast growth factor 23 regulates renal 1,25-dihydroxyvitamin D and phosphate metabolism via the MAP kinase signaling pathway in Hyp mice. J Bone Miner Res. 2011;26(8):1883-1890.

19. Zhang MY, Ranch D, Pereira RC, Armbrecht HJ, Portale AA, Perwad F. Chronic inhibition of ERK1/2 signaling improves disordered bone and mineral metabolism in hypophosphatemic (Hyp) mice. Endocrinology. 2012;153(4):1806-1816.

20. Isnardi I, et al. Complement receptor 2/ CD21- human naive B cells contain mostly autoreactive unresponsive clones. Blood. 2010;115(24):5026-5036.

21. Ferre EM, et al. Redefined clinical features and diagnostic criteria in autoimmune polyendocrinopathy-candidiasis-ectodermal dystrophy. JCI Insight. 2016;1(13):e88782.

22. Warnatz K, et al. Expansion of CD19(hi)CD21(lo/ neg) B cells in common variable immunodeficiency (CVID) patients with autoimmune cytopenia. Immunobiology . 2002;206(5):502-513.

23. Kuehn HS, et al. Immune dysregulation in human subjects with heterozygous germline mutations in CTLA4. Science. 2014;345(6204):1623-1627.

24. Alon U, Hellerstein S. Assessment and interpretation of the tubular threshold for phosphate in infants and children. Pediatr Nephrol. 1994;8(2):250-251. 